\title{
Consideraciones para la aplicación del método Coral Tree Nursery en la recuperación de poblaciones de Acropora valida (Acroporidae) en dos zonas neríticas de Galápagos, Ecuador
}

\section{Considerations for the application of the Coral Tree Nursery method in the recovery of populations of Acropora valida (Acroporidae) in two neritic zones of Galapagos, Ecuador}

Natalí Galárraga G.

Universidad Internacional del Ecuador, Ecuador

Patricio Yánez M.

Universidad Internacional del Ecuador, Ecuador

Universidad Iberoamericana del Ecuador, Ecuador

Universidad del Zulia, Venezuela

Autores para correspondencia: natygalarragag@hotmail.com; apyanez@ hotmail.com Fecha de recepción: 20 de julio de 2018 - Fecha de aceptación: 15 de septiembre de 2018

Resumen: el presente documento describe diferentes aspectos técnicos que se deben considerar para implementar el método de reproducción asexual Coral Tree Nursery y efectuar el seguimiento de su grado de éxito al trabajar con la recuperación de poblaciones del coral Acropora valida, presente en zonas coralinas de aguas neríticas de las Islas Galápagos. Se analiza el contexto teórico del desarrollo de este método, así como la descripción física del mismo, las zonas de Galápagos en las cuales podría ser aplicado y la forma de seguimiento y análisis técnico de la información relacionada con la supervivencia, crecimiento y prendimiento de los fragmentos de coral incluidos en los viveros propuestos.

Palabras Clave: Islas Galápagos; corales; Acropora valida; zona nerítica; coral tree nursery

Abstract: this article describes different technical aspects that should be considered to implement the Coral Tree Nursery asexual reproduction method and monitor its degree of success in working with the recovery of Acropora valida coral populations, present in coral reef areas of the Galapagos Islands. The theoretical context of the development of this method is analyzed, as well as its physical description, the areas of Galapagos in which it could be applied and the ways for monitoring. Finally, we propose some technical analysis for the information related to the survival, growth and transplantation of fragments of coral included in the proposed nurseries.

Key Words: Galapagos Islands; corals; Acropora valida; neritic zone; coral tree nursery 


\section{Introducción}

Los arrecifes de coral tienen una gran importancia como ecosistemas marinos, constituyen zonas de alta biodiversidad, así como lugares de criadero de algunas especies de peces y de invertebrados variados (Reaka-Kudla et al., 1997; Yánez, 2014).

Los arrecifes de corales mantienen cerca del $10 \%$ del total de peces consumidos por los humanos, y se estima que los ecosistemas de los arrecifes de corales generan anualmente algo más de US\$ 100 mil millones para los pesqueros del mundo (Camargo et al., 2009). El valor total de todos los bienes y servicios que proveen los arrecifes de corales ha sido estimado en US\$ 375 mil millones por año (Edwards \& Gómez 2007). A finales del siglo XX se estimó que el $70 \%$ de los arrecifes de corales del mundo se encuentran directamente amenazados por actividades humanas (Hughes et al., 2003).

A nivel regional y mundial, los corales de las Islas Galápagos son relativamente bien conocidos gracias al interés general que existe por estas islas y su entorno. A pesar de ello, estos arrecifes coralinos están desapareciendo y la mayoría de sus comunidades se han degradado severamente después del evento de El Niño (ENSO) de 1982 - 1983; algunos autores mencionan que la cobertura total de corales se redujo como consecuencia de ésto en un 95 al $99 \%$ a fines del siglo XX (Glynn, 2003).

Galápagos es el hogar de 25 especies endémicas de peces, moluscos, crustáceos, equinodermos, corales y macroalgas que cumplen con los criterios para especies amenazadas, debido a la disminución de la extensión de su ocurrencia o sus rangos altamente localizados y que, por lo tanto, califican para su inclusión en la Lista Roja de la UICN. Sin dejar de lado que los corales proveen además de refugio a numerosas especies marinas amenazadas, incluyendo 16 mamíferos, aves, reptiles y peces, actualmente también reconocidos en la Lista Roja de Especies Amenazadas de la UICN de 2006 (Graham et al., 2008). Por lo tanto, la protección y conservación de los arrecifes coralinos de Galápagos es de suma importancia.

Acropora valida (Acroporidae) (Figura 1) es una especie de coral que se encuentra distribuida en la costa continental ecuatoriana desde la provincia de Esmeraldas hasta la costa de Las Tunas en la provincia de Manabí y en todo el Archipiélago de Galápagos (Richards et al., 2014). Las especies del género Acropora suelen jugar un papel fundamental como un componente estructural clave de los arrecifes de coral y contribuyen a la complejidad del entorno del arrecife, creando un hábitat vital y mayor disponibilidad de nichos (Gratwicke \& Speight, 2005).

Varios factores han afectado directa o indirectamente a los corales en los últimos años, puesto que son muy sensibles a cambios bruscos de factores abióticos; las actividades humanas también incrementan el debilitamiento y la mortalidad de los corales (Hughes et al., 2003). Hay evidencias que apuntan a que la mayor tasa de mortalidad de corales se encuentra relacionada al calentamiento anormal de las aguas (Colgan, 1990), por ejemplo. 


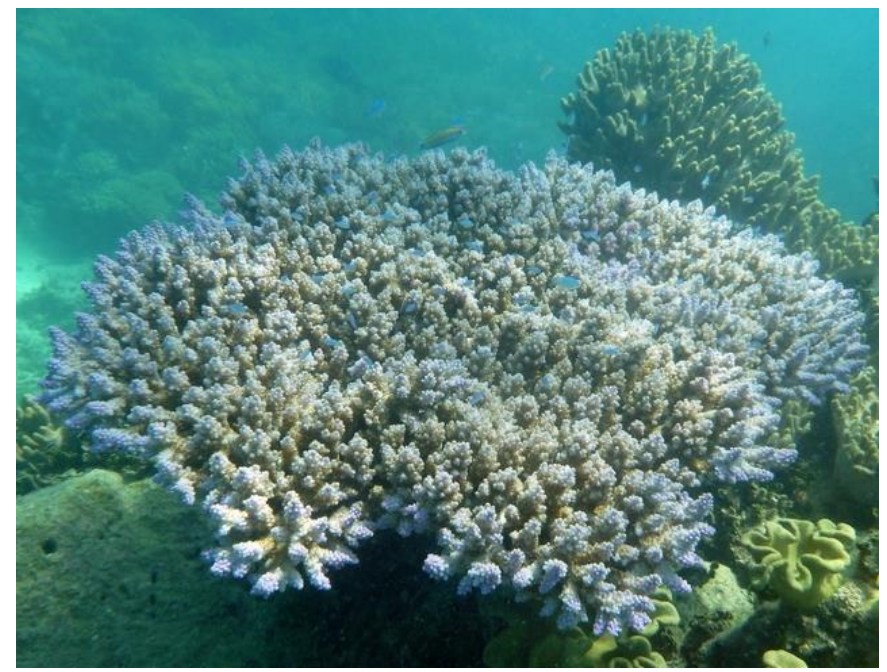

Figura No. 1. Pólipo de Acropora valida.

Fuente: Witherall \& Lyka, http://www.gaiaguide.info/HotShot.html?resourceId=Sxf5HINK,

Acropora tiene una recuperación natural limitada (Kojis \& Quinn, 2001) debido a tasas bajas de reclutamiento sexual, baja supervivencia del recluta y poca conectividad (Schopmeyer et al., 2012). Mediante la reproducción asexual por medio de fragmentación (Wallace, 1985) pueden llegar a formar colonias enteras a partir de pequeños brazos de corales (Connell, 1973). Los viveros de corales mediante la reproducción asexual y rápido crecimiento de los Acropóridos (Soong \& Chen, 2003) hacen factible el "trasplante" por medio de fragmentos de coral (Alcalá et al., 1982; Guzmán, 1999).

Los arrecifes submareales rocosos de Galápagos constituyen el hábitat de mayor ocurrencia, cobertura y distribución en las Islas Galápagos (Wellington, 1984), en donde las operaciones pesqueras se realizan con mayor intensidad, consecuentemente, éstos han disminuido paulatinamente en las últimas décadas (Graham et al., 2002).

Los eventos de El Niño (ENSO) por lo general tienen efectos devastadores sobre los corales llevándolos al blanqueamiento y posteriormente a la muerte, pudiendo tener como consecuencia la apertura de nichos que especies invasoras ocupan en menos tiempo que el que las especies nativas necesitan para recuperarse (Keith et al., 2015). Las interacciones significativas entre la sobrepesca y el ENSO pueden ser importantes para determinar el riesgo de extinción de los corales (Glynn, et al., 2017). De igual manera, las tasas de crecimiento lento del coral y la interdependencia de las especies de arrecifes submareales sugieren que los efectos de la rápida mortalidad de los corales podrían propagarse rápidamente en toda la comunidad, causando cambios significativos en el equilibrio del ecosistema (Banks et al., 2009).

\section{La necesidad de contar con estrategias de repoblación de los corales en general y de Acropora valida en particular}

Los arrecifes de coral son centros de alta biodiversidad, proporcionan un hábitat esencial para muchas especies de importancia comercial, como peces e invertebrados, y ofrecen protección costera; a pesar de que representan sólo un pequeño porcentaje, menos del 1\% del medio marino (Verón et al., 2009). Los arrecifes de coral protegen las costas de 100 países 
(Moberg \& Folke, 1999), evitando la erosión costera, protegiendo manglares y hábitats de lagunas salobres que apoyan algunas etapas de la vida de importantes especies marinas (Marshall \& Johnson, 2007). Estos frágiles ecosistemas también brindan recursos pesqueros que proporcionan una importante fuente de alimento para los seres humanos y gracias a su belleza natural generan también miles de millones de dólares por turismo cada año (Johnson et al., 2011).

Ante la perspectiva de la recuperación natural limitada debido a las tasas bajas de reclutamiento sexual, baja supervivencia de los juveniles y eventos reproductivos y liquidación altamente variables (Kojis \& Quinn, 2001), se está recurriendo a la restauración como un mecanismo potencial para mitigar el descenso de los patrones y aumentar el potencial de recuperación de las poblaciones de corales dañadas o empobrecidas (Edwards \& Gómez, 2007).

Debido a la recuperación natural lenta de los arrecifes y su alto potencial económico, se han propuesto diversos métodos para la reconstrucción del arrecife coralino y se han hecho esfuerzos para facilitarla (Guzmán, 1999). Mediante la propagación de especies de Acropora que tienen el potencial para llevarse a cabo con éxito a bajo costo, utilizando diferentes técnicas de viveros, se puede ayudar a las comunidades costeras locales para restaurar y gestionar sus propios recursos en los arrecifes locales (Young et al., 2012).

La tendencia actual de la población de corales es hacia la disminución, entre las medidas recomendadas para la conservación se encuentra la gestión de recuperación de las especies; medidas de restauración, propagación y el uso de técnicas artificiales pueden llegar a ser importantes para la conservación de las especies dentro del género Acropora (Richards et al., 2014). La conservación futura de Acropora spp. Mediante métodos de restauración innovadores es de gran importancia (Bliss, 2015). Acropora es más resistente a algunas de las amenazas que enfrentan los corales y, por tanto, la disminución de su población se estima solo mediante el porcentaje de arrecifes destruidos; a pesar de su mayor resistencia, la amenaza más importante para Acropora es la reducción y pérdida del hábitat de arrecifes (Wilkinson, 2004). Las especies de corales de Acropora han sido de gran importancia para la evolución, biogeografía y ecología de los arrecifes de coral del mundo (Wallace et al., 2012), con aproximadamente 150 especies existentes en su mayoría en el Indo-Pacífico y algunas en el Caribe (Wallace, 1999), es considerado el género con mayor abundancia y diversidad de especies en el Pacífico (Wallace \& Rosen, 2006). Este género es capaz de reproducirse asexualmente por fragmentación y su rápido crecimiento en comparación a otros géneros (Prahl \& Mejia, 1985) podría facilitar su conservación.

Los acropóridos y otras especies de corales ramificados son buenos candidatos para los viveros de corales por su habilidad de reproducción asexual y su rápido crecimiento (Soong \& Chen, 2003). Cada vivero apunta al crecimiento, con eficiencia y eficacia, de los corales que se replantarán al entorno natural del arrecife (Bliss, 2015). La metodología de vivero de coral ha demostrado ser una herramienta viable para el cultivo de fragmentos de Acropora en todo el Caribe (Johnson et al., 2011), siendo un componente esencial en los actos de restauración de arrecifes promoviendo la diversidad genética y aumentando el éxito reproductivo (Shafir et al., 2006, Nedimyer et al., 2011). 


\section{Métodos de repoblación del coral Acropora valida}

La producción de corales del género Acropora mediante viveros se ha llevado a cabo en algunos países del Caribe y en el Atlántico occidental con el fin de restaurar los arrecifes de corales (Young et al., 2012). Nedimyer de la Fundación Restauración de Corales y William Precht de la NOAA (2010) realizaron viveros con Acropora palmata en los Cayos de Florida y en parte del Atlántico occidental tropical teniendo resultados satisfactorios con la creación de nuevas colonias mediante la fragmentación.

Soong y Chen (2003) realizaron una transplantación de Acropora pulchra al sur de Taiwán mediante la regeneración y crecimiento de esta especie en vivero, teniendo éxito con los fragmentos de coral en un vivero semi protegido. En 2012, Young y colaboradores realizaron una revisión de las restauraciones de los arrecifes de coral y su propagación. En 2015, Bliss realizó varios análisis de la traslocación de Acropora en varias zonas de Florida y reporta un 92\% de éxito en las colonias reubicadas; dando a conocer que tal supervivencia en Florida fue alta, proporcionando soporte para el intercambio de colonias entre viveros, que se beneficiarán en gran medida de los esfuerzos de restauración de colaboración.

Un método bastante utilizado para el crecimiento de coral y su restauración implica suspender los corales sobre el fondo del mar retorciendo el fragmento de coral en cuerdas, o colgar el fragmento de coral de los cables, o cuerdas atadas a líneas horizontales apiladas (Bowden, 2001; Quinn, 2005). Estos métodos permiten que los corales cuelguen en el agua y crezcan en todas las direcciones. Los requisitos de mantenimiento para este método se reducen considerablemente, y las tasas de crecimiento y supervivencia son positivamente altas. Sin embargo, existe riesgo de enredo de especies marinas principalmente tortugas marinas, y son propensos a la flacidez y enredos en el proceso de crecimiento y ganancia de masa de los corales (Nedimyer et al., 2011).

Otro método común de cultivo de corales in situ implica la fragmentación de un pequeño trozo de coral, entre 5 a $10 \mathrm{~cm}$ de longitud, y adjuntar el coral en un disco redondo con cable o cuerda (Ellis \& Ellis, 2001). El disco se une a un soporte que por lo general está levantado del suelo marino usando algún tipo de material fácilmente disponible, tales como barras de refuerzo de acero o de bloques de hormigón. Este método es laborioso de configurar y mantenerlo, y es vulnerable a las perturbaciones climáticas y la depredación.

El Coral Tree Nursery es un marco simple con diferentes niveles que se asemeja a la forma de un árbol. Se utiliza fragmentos de corales como en los métodos anteriores, pero éstos están simplemente unidos a los brazos del árbol utilizando alambre o monofilamento. Este marco permanece amarrado al fondo del mar y se mantiene estable con un dispositivo de flotación debajo del agua que se puede mover hacia arriba y abajo de la columna de agua en caso de necesidad. El árbol flota en la columna de agua y es capaz de moverse con la onda generada por mareas de tempestad. Esto disipa la energía de onda previniendo de daños a la estructura de árbol o los propios corales. El mantenimiento se reduce significativamente ya que el área superficial para el crecimiento de algas nocivas es limitada (Nedimyer et al., 2011). 
Con el proceso de fragmentación se puede producir múltiples fragmentos desde un pequeño producto de inicio (Johnson et al., 2011). Mediante el método Coral tree Nursery se propone, a través del presente documento, estimular la realización futura de viveros en dos zonas neríticas en Galápagos utilizando Acropora valida, buscando la restauración y rehabilitación de los arrecifes de coral locales.

\section{Consideraciones para la aplicación del método Coral Tree Nursery en procesos de repoblación de Acropora valida}

Un estudio como el propuesto debe girar en torno a los siguientes objetivos:

\section{General}

- Evaluar la eficiencia del método Coral Tree Nursery para el crecimiento en vivero de Acropora valida en dos zonas de Galápagos.

\section{Específicos:}

- Evaluar la tasa de supervivencia del coral Acropora valida en el vivero.

- Determinar valores de crecimiento de Acropora valida en el vivero.

- Identificar los mejores sitios dentro del área meta para la creación de viveros para fomentar la conservación de corales de Acropora valida.

\section{Área propuesta para la repoblación}

Galápagos se encuentra ubicado a 960 kilómetros del Ecuador continental, en el océano Pacífico (Figura 2). Siendo la primera área protegida ecuatoriana declarada en 1934, cuenta actualmente con el 95\% de su área total protegida; se caracteriza por tener un alto grado de endemismo de especies de flora y fauna (SPNG \& MAE, 2005).

Las Islas Galápagos se benefician de la Corriente Ecuatorial Sur (CES) que fluye hacia el oeste en su paso hacia el Pacífico central, ésta varía estacionalmente en magnitud y en latitud. En los años neutrales en los que las islas no tienen contacto con ENSO, las islas del norte Darwin y Wolf se caracterizan por tener aguas más cálidas, resultado de la desviación del sudoeste de la Contracorriente Ecuatorial del norte (NECC) que se extiende desde el Golfo de Panamá. Las Islas Darwin y Wolf se encuentran separadas de 1 a $2^{\circ}$ de latitud de las islas centrales teniendo a su vez como resultado $1-2^{\circ} \mathrm{C}$ aguas marinas más calientes durante todo el año (Glynn et al., 2017). 


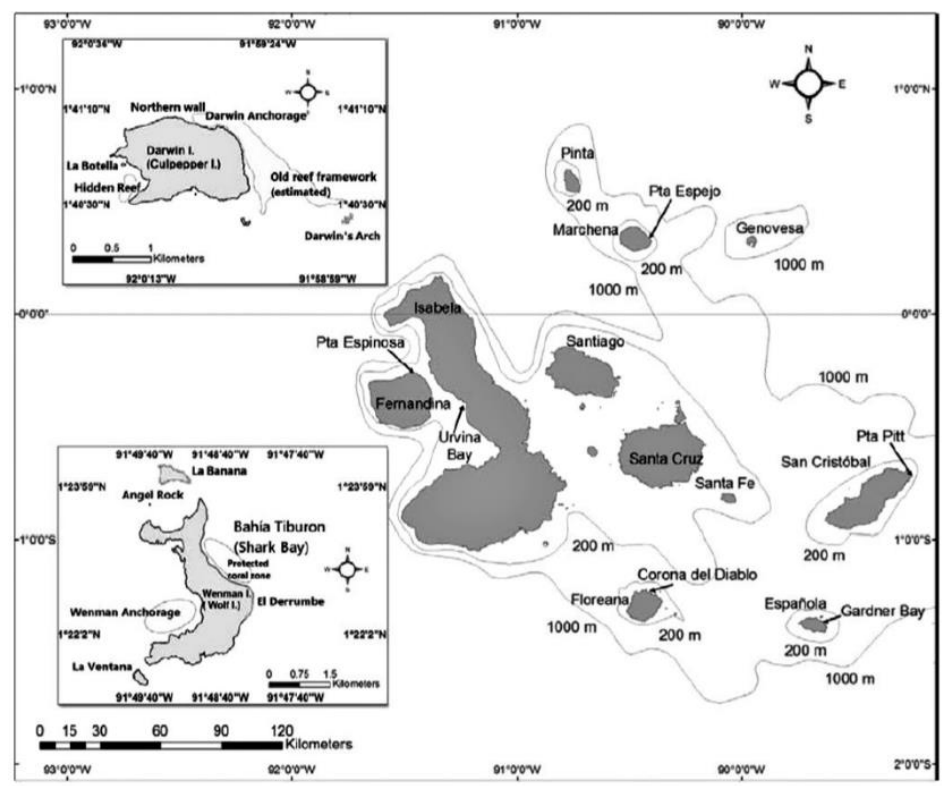

Figura No. 2. Islas Galápagos, Ecuador. A la izquierda las dos zonas geográficas propuestas para la investigación: islas Darwin (Culpepper) y Wenman (Wolf), ubicadas entre $1^{\circ} 21^{\prime} \mathrm{N}$ y $1^{\circ} 41^{\prime} \mathrm{N}$.

Fuente: Glynn et al., 2017.

\section{El inicio del proceso}

Cerca de la Isla Darwin se recomienda tomar muestras de Acropora valida dentro del arrecife Wellington (Figura 3), colectando fragmentos de corales considerando la presencia de diferentes fenotipos para garantizar una mayor diversidad morfológica.

De igual manera, cerca de la Isla Wolf se recomienda realizar la toma de muestras (fragmentos) de Acropora valida dentro de la comunidad de coral ubicada a lo largo de la plataforma noreste en Shark Bay (Figura 4), provenientes de distintos fenotipos de esta especie.

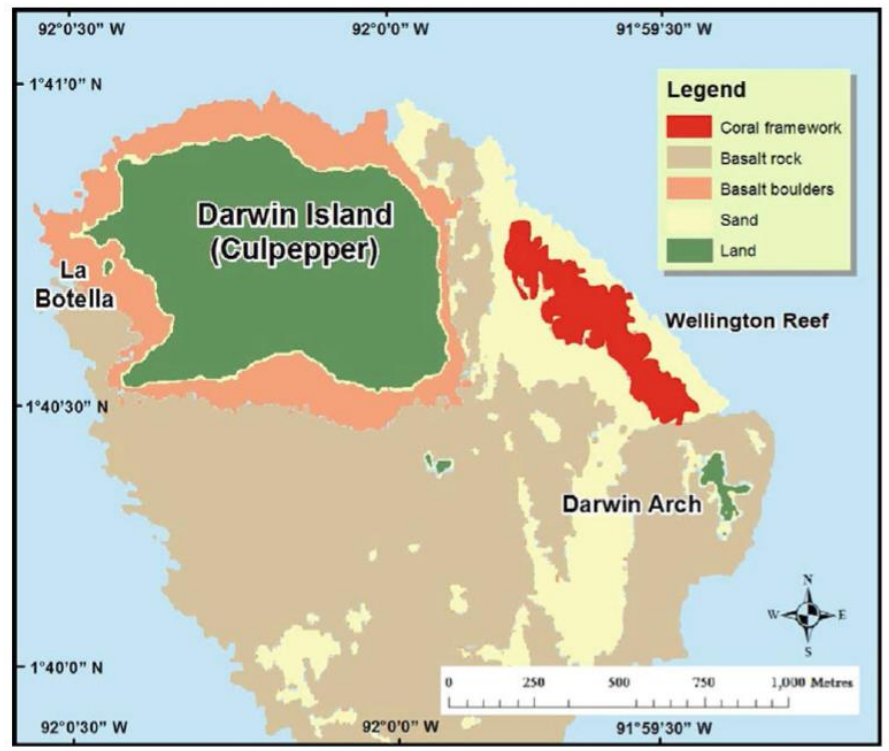

Figura No. 3. Ubicación del arrecife Wellington en relación a la Isla Darwin. Fuente: Glynn et al., 2017. 


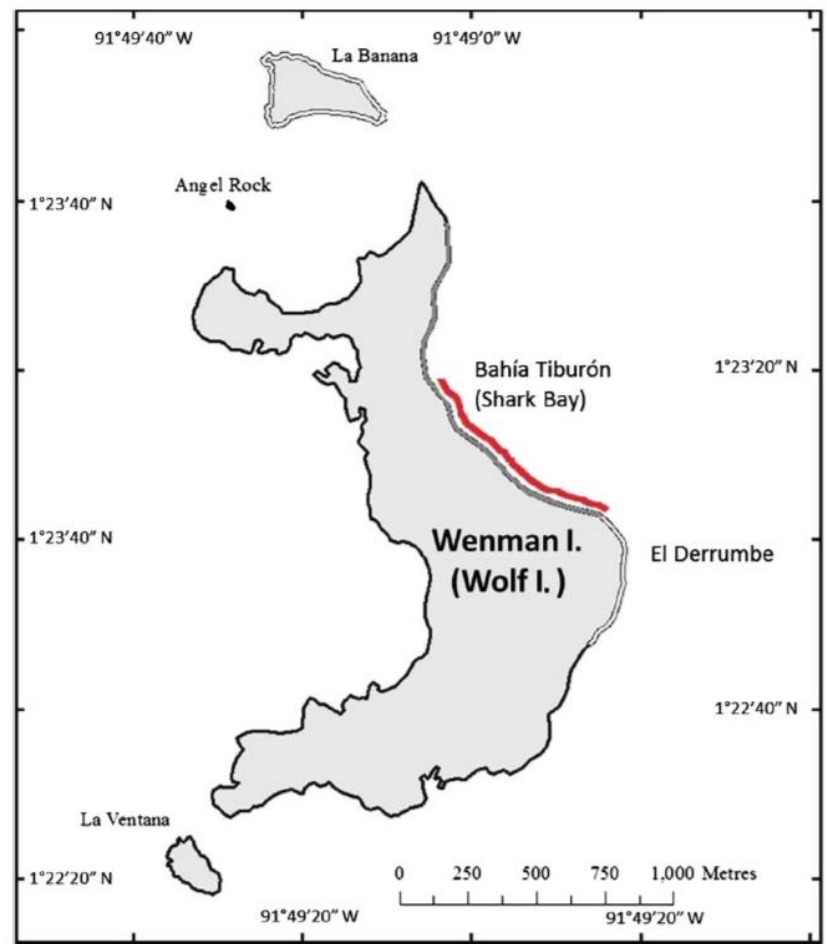

Figura No. 4. Comunidad de coral (línea roja) en Shark Bay, muy cerca de la Isla Wolf. Fuente: Glynn et al., 2017.

\section{Instalación y delimitación de los viveros}

De manera inicial se deberá realizar un recorrido de reconocimiento dentro del arrecife y $100 \mathrm{~m}$ a la redonda del mismo para delimitar condiciones locales y zonas en las que serán instalados los viveros; se colocarán 3 viveros por zona.

Cada punto para la instalación de un vivero se encontrará distanciado por 5 metros del siguiente más cercano en la zona, todos los viveros se encontrarán a una distancia máxima de 50 metros del arrecife para contar con un mejor manejo de traslado de fragmentos hacia el vivero.

Se colocarán los viveros Coral Tree Nursery en los puntos respectivos, posteriormente se deberán tomar los fragmentos de los brazos de corales con un diseño de fragmentación de máximo el $75 \%$ del brazo, teniendo en cuenta que se encuentren representados diferentes fenotipos en la colecta (Figura 5) para ser colocados en los viveros (80 fragmentos por vivero).

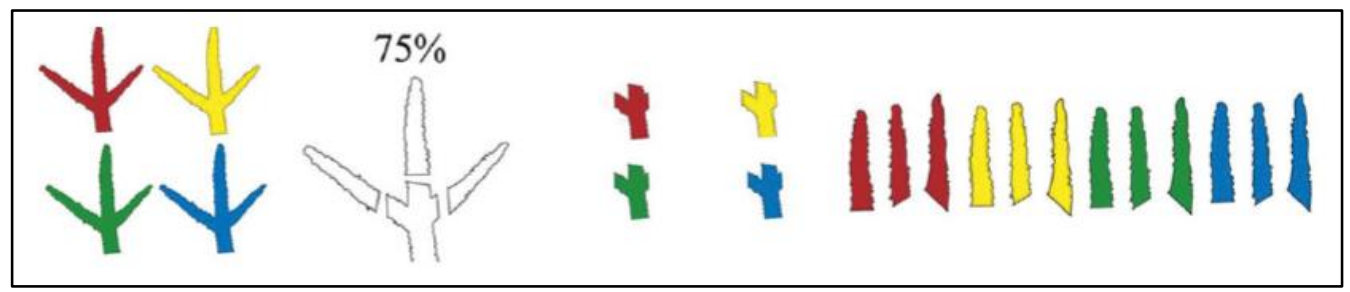

Figura No. 5. Método de fragmentación y replicación al 75\%, tomando en cuenta que cada color es un fenotipo diferente. Fuente: Adaptado de Lohr et al., 2015. 
Tipo de Vivero propuesto (considerando a Nedimyer et al., 2011)

En cada punto seleccionado se deberá instalar un Coral Tree Nursery, compuesto por un marco hecho con tubos de PVC y 20 brazos individuales de 0,5 metros separados por $13 \mathrm{~cm}$ de distancia entre sí (Figuras 6 y 7). Para garantizar la estabilidad de cada vivero individual, el mismo se amarrará en el fondo a una estaca y se colocará un flotador debajo de la superficie del agua, mismo que mantendrá a flote al vivero (Figura 7). Los fragmentos de corales se sujetarán a los brazos de la estructura con alambre o monofilamentos.

Se recomienda que el marco tenga una columna de 1,3 metros de longitud. Cada brazo de la estructura podrá sostener 4 fragmentos de corales, separados por $10 \mathrm{~cm}$ uno del otro.
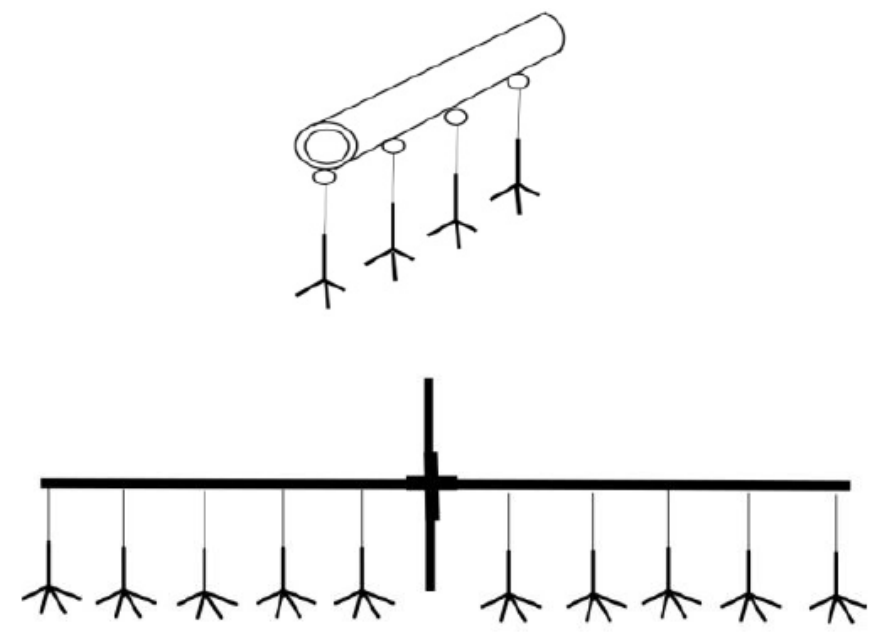

Figura No. 6. Suspensión del coral en brazos de la estructura. Fuente: Nedimyer et al., 2011.

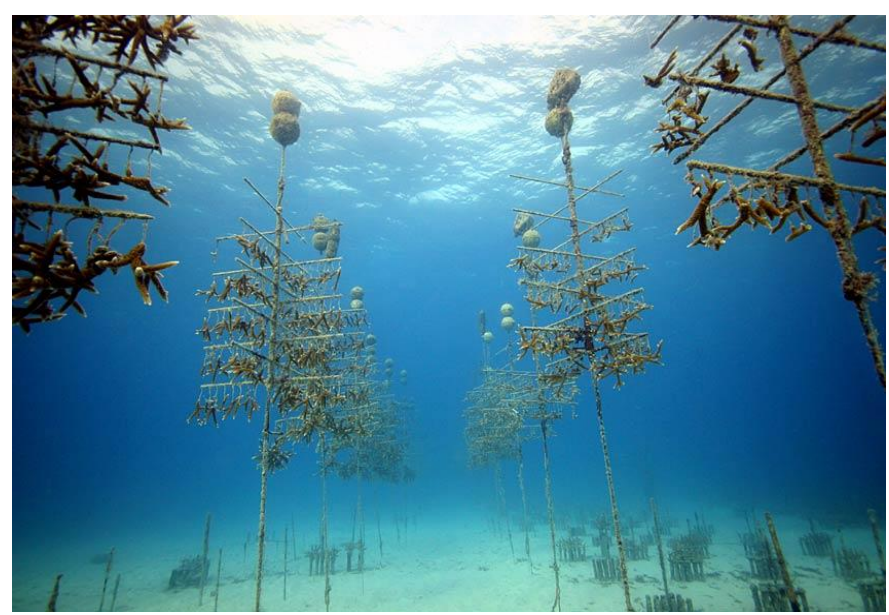

Figura No. 7. Modelo posible para un Coral Tree Nursery. Fuente: https://blissology.com/wp-content/uploads/2017/08/ 


\section{Seguimiento a la supervivencia, crecimiento y replantación}

Para el registro y organización de información concerniente a las etapas de desarrollo del coral se deberán considerar los siguientes ámbitos:

\section{En torno a la etapa de crecimiento}

Los corales deberán cultivarse en el vivero durante aproximadamente cuatro meses. En este lapso se deben efectuar visitas periódicas a cada vivero, habiendo codificado y etiquetado previamente a cada fragmento y procediendo al seguimiento de la supervivencia y valores de crecimiento de los corales una vez por semana.

Cada vivero y fragmento de coral además contará con información adicional como coordenadas geográficas, profundidad, posición, características del ambiente local y otras necesarias para el estudio.

\section{En torno a la replantación}

Al no saber el valor exacto del tamaño competente para el trasplante de un coral Acropora valida, ya que la investigación propuesta sería pionera con esta especie, se recomienda que los corales sean retirados del Coral Tree Nursery 24 meses después de haber sido colocados inicialmente en él (considerando lo propuesto por Nedimyer et al., 2011). Los individuos ya retirados volverán a ser etiquetados y llevados al arrecife coralino del que se tomó el fragmento (o a otro cercano considerando la opinión de expertos), al que deberán unirse bajo el agua utilizando un adhesivo.

Con este nuevo etiquetado durante la replantación, se podrá mantener de igual manera un seguimiento del estado de cada coral posterior a la replantación (Nedimyer et al., 2011).

\section{Recomendaciones para el tratamiento y análisis de la información}

La tasa de crecimiento de Acropora valida suele ser de 0,80 mm por mes (Harriot, 1999), con lo que se esperaría tener un crecimiento cercano a los 3,2 $\mathrm{mm}$ al cabo de cuatro meses. Es importante recalcar que esta tasa de crecimiento se la ha estudiado en el este australiano, teniéndola como referencia más cercana para el presente trabajo, al no contar con datos referenciados en literatura especializada sobre el Pacífico oriental.

\section{Supervivencia}

Con los datos registrados en campo, será necesario efectuar análisis y curvas de supervivencia de los corales por cada mes de avance del proceso: tanto durante la fase de vivero como durante la fase inmediatamente posterior a la replantación. Con esta información se podrá contar con índices de supervivencia, de mortalidad y de prendimiento exitoso. Estos índices podrán permitir generar información sobre la viabilidad del proceso propuesto.

\section{Tasa de crecimiento}


Mediante la toma de datos de evolución del tamaño de los fragmentos, se procederá a determinar la tasa de crecimiento mensual de cada fragmento de coral dentro del vivero mediante la fórmula:

Porcentaje de crecimiento $=(($ tamaño final - tamaño anterior $) /$ tamaño anterior $)$

Con los datos individuales de crecimiento se logrará obtener un promedio total del crecimiento a lo largo del vivero.

\section{Tasa de mortalidad}

De igual manera, con los datos obtenidos sobre mortalidad en el vivero, se procederá a determinar tasas mensuales de supervivencia y de mortalidad de los fragmentos de coral dentro de los viveros, aplicando la siguiente fórmula:

Tasa de mortalidad:(No. de fragmentos muertos en la población / Población total inicial) x 100

\section{Otros análisis}

Análisis estadísticos descriptivos e inferenciales (univariados o multivariados) podrán también ser aplicados a la información generada en el estudio propuesto, considerando las variables de interés y las hipótesis examinadas, de acuerdo a lo propuesto por Yánez (1997, 2005). Por ejemplo, análisis de varianza paramétricos o no paramétricos podrían arrojar conclusiones importantes sobre la existencia o no de diferencias significativas en la supervivencia de individuos y/o en el crecimiento de los mismos en relación a las características de los sitios de colocación de los viveros.

\section{Bibliografía}

Alcalá, A., Gómez, E., \& Alcalá, L. (1982). Survival and growth of coral transplants in central Philippines. Kalikasan Philippine Journal of Biology, 11, 136-147.

Banks, S., Vera, M., \& Chiriboga, A. (2009). Establishing reference points to assess long-term change in zooxanthellate coral communities of the northern Galápagos coral reefs. Galapagos Research, 66, 43-64.

Bliss, B. (2015). Translocation of Acropora cervicornis across Geographic Regions: Investigating Species Recovery and Restoration. Florida, Master's thesis. Nova Southeastern University. Retrieved from NSUWorks, Oceanographic Center.

(30). http://nsuworks.nova.edu/occ_stuetd/30.

Bowden, W. (2001). Coral transplantation modeled after natural fragmentation processes: lowtech tools for coral reef restoration and management. Mayaguez, PhD thesis, University of Puerto Rico.195. 
Camargo, C., Maldonado, J., Alvarado, E., Moreno-Sánchez, R., Mendoza, S., Manrique, N., \& Sánchez, J. (2009). Community involvement in management for maintaining coral reef resilience and biodiversity in southern Caribbean marine protected areas. Biodiversity and Conservation, 18(4), 935-956.

Colgan, M. (1990). El Niño and the history of eastern Pacific reef building. Elsevier Oceanography Series, 52, 183-232.

Connell, J. (1973). Population ecology of reef building corals. En: Jones, O., \& Endeans, R. (Eds.). Biology and geology of coral reefs. London, Academic Press. Pp. 205- 245.

Edwards, A., \& Gómez, E. (2007) Reef restoration concepts and guidelines: making sensible management choices in the face of uncertainty. Building for Management Programme/Coral Reef Targeted Research 38.

Ellis, S., \& Ellis, E. (2001). Recent Advances in Lagoon-based Farming Practices for Eight Species of Commercially Valuable Hard and Soft Corals - A Technical Report. Waimanalo, CTSA Publication No. 147.63pp.

Glynn, P. (2003). Coral communities and coral reefs of Ecuador. En: Latin American coral reefs. Amsterdam, Elsevier, 449-472.

Glynn, P., Alvarado, J., Banks, S., Cortés, J., Feingold, J., Jiménez, C., \& Navarrete, S. (2017). Eastern Pacific coral reef provinces, coral community structure and composition: an overview. En: Coral Reefs of the Eastern Tropical Pacific. Netherlands, Springer. 7. Pp. 107-176.

Graham, E., Fariña, J., Calvopiña, M., Martínez, C., \& Banks, S., (2002). Comunidades submareales rocosas ii: peces y macroinvertebrados móviles. En: Danulat, E., \& Edgar, G. (Eds.). Reserva Marina de Galápagos. Línea Base de la Biodiversidad. Fundación Charles Darwin/Servicio Parque Nacional Galápagos, Santa Cruz, Galápagos. Pp. 68-92.

Graham, E., Banks, S., Bensted-Smith, R., Calvopiña, M., Chiriboga, A., Garske, L. \& Salazar, S. (2008). Conservation of threatened species in the Galapagos Marine Reserve through identification and protection of marine key biodiversity areas. Aquatic Conservation: Marine and Freshwater Ecosystems, 18(6), 955-968.

Gratwicke, B., \& Speight, M. (2005) The relationship between fish species richness, abundance and habitat complexity in a range of shallow tropical marine habitat. Journal of Fish Biology, 66, 650-665.

Guzmán, H. (1999). Large-scale restoration of Eastern Pacific reefs: the need for understanding regional biological processes. Fort Lauderdale, Florida: National Coral Reef Institute, Nova Southeastern University.

Harriott, V. (1999) Coral growth in subtropical eastern Australia. Coral Reefs, 18, 281-291. 
Hughes, T., Baird, A., Bellwood, D., Card, M., Connolly, S., Folke, C., \& Lough, J. (2003). Climate change, human impacts, and the resilience of coral reefs. Science, 301(5635), 929-933.

Johnson M., Lustic, C., \& Bartels, E. (2011) Caribbean Acropora restoration guide: Best practices for propagation and population enhancement. The Nature Conservancy. 54pp.

Keith, I., Dawson, T., Collins, K., \& Bank, S. (2015). Especies marinas invasoras en la Reserva Marina de Galápagos: Un caso para investigación adicional, mejoramiento del manejo y revisión de políticas. En: Informe Galápagos 2013-2014. DPNG, CGREG, FCD y GC. Puerto Ayora, Galápagos. Pp. 83-88.

Kojis, B., \& Quinn, N. (2001). The importance of regional differences in hard coral recruitment rates for determining the need for coral restoration. Bull Mar Sci., 69, 967-974.

Lohr, K., Bejarano, S., Lirman, D., Schopmeyer, S., \& Manfrino, C. (2015). Optimizing the productivity of a coral nursery focused on staghorn coral Acropora cervicornis. Endangered Species Research, 27(3), 243-250.

Marshall, P., \& Johnson, J. (2007). The Great Barrier Reef and climate change: vulnerability and management implications. En: Reef Marine Park Authority and the Australian Greenhouse Office. Climate change and the Great Barrier Reef. Australia, Pp.: 774-801.

Moberg, F. \& Folke, C. (1999). Ecological goods and services of coral reef ecosystems. Ecological Economics, 29, 215-233.

Nedimyer, K., Grablow, K., Northrop, A., \& Precht, W. (2010). Producing Acropora palmata in offshore coral nurseries for reef restoration. En: Linking Science to ManagementConference and Workshop on the Florida Keys Marine Ecosystem. Duck Key, FL. 128.

Nedimyer K., Gaines K., \& Roach S., (2011). Coral Tree Nursery@: An innovative approach to growing corals in an ocean-based field nursery. AACL Bioflux, 4(4), 442-446.

SPNG \& MAE: Servicio del Parque Nacional Galápagos y Ministerio del Ambiente del Ecuador. (2005). Plan de Manejo del Parque Nacional Galápagos, Parque Nacional Galápagos y Ministerio del Ambiente del Ecuador, Puerto Ayora, Galápagos, Ecuador.

Prahl, H., \& Mejia, A. (1985). Primer informe de un coral acropórido, Acropora valida Dana, 1846 (Scleractinia: Astrocoeniida: Acroporidae) para el Pacífico americano. Revista de Biología Tropical, 33, 39-43.

Quinn, N. (2005). Coral Reef Gardening Project under USAID's Coastal Water Quality Improvement Project Phase II (CWIP2) Final Report, Florida, Discovery Bay Marine Laboratory, UWI 20 pp. 
Reaka-Kudla, M., Wilson, D., \& Wilson, E. (Eds.). (1997). Biodiversity II: understanding and protecting our biological resources. Washington, DC: Joseph Henry Press.

Richards, Z., Delbeek, J., Lovell, E., Bass, D., Aeby, G. \& Reboton, C. (2014). Acropora valida. The IUCN Red List of Threatened Species 2014: e.T133293A54231035. http://dx.doi.org/10.2305/IUCN.UK.2014-1.RLTS.T133293A54231035.en

Schopmeyer, S., Lirman, D., Bartels, E., Byrne, J., Gilliam, D., Hunt, J., \& Walter, C. (2012). In situ coral nurseries serve as genetic repositories for coral reef restoration after an extreme cold-water event. Restoration Ecology, 20(6), 696-703.

Shafir, S., Van Rijn, J., \& Rinkevich, B. (2006). Steps in the construction of underwater coral nursery, an essential component in reef restoration acts. Marine Biology, 149(3), 679-687.

Soong, K., \& Chen, T. (2003). Coral transplantation: regeneration and growth of Acropora fragments in a nursery. Restoration Ecology, 11(1), 62-71.

Tree Nursery for Staghorn Coral. (2017). Disponible en: https://blissology.com/wpcontent/uploads/2017/08/tree-nursery-for-staghorn-coral-2.jpg. Fecha de consulta: 10 de enero de 2018.

Verón, J., Hoegh-Guldberg, O., Lenton, T., Lough, J, Obura, D., Pearce-Kelly, P., \& Rogers, A. (2009). The coral reef crisis: The critical importance of $<350 \mathrm{ppm} \mathrm{CO}_{2}$. Marine pollution bulletin, 58(10), 1428-1436.

Wallace, C. (1985). Reproduction, recruitment and fragmentation in nine sympatric species of the coral genus Acropora. Marine Biology, 88, 217-233.

Wallace, C. (1999). Staghorn corals of the world: a revision of the genus Acropora. CSIRO Publishing.

Wallace, C., \& Rosen, B. (2006). Diverse staghorn corals (Acropora) in high-latitude Eocene assemblages: Implications for the evolution of modern diversity patterns of coral reefs. Proceedings of the Royal Society of London B: Biological Sciences, 273(1589), 975-982.

Wallace, C., Done, B. \& Muir, P. (2012). Revision and catalogue of worldwide staghorn corals Acropora and Isopora (Scleractinia: Acroporidae) in the Museum of Tropical Queensland. Memoirs of the Queensland Museum - Nature 57: 1-255.

Wellington, G. 1984. Marine environment and protection. En: Perry, R. (Ed.). Key Environments: Galápagos. Oxford: Pergamon Press. Pp. 247-263.

Witherall, D., \& Lyka, Imagen de Acropora valida. Disponible en: http://www.gaiaguide.info/HotShot.html?resourceId=Sxf5HINK, Fecha de consulta: 10 de enero de 2018. 
Wilkinson, C. (2004). Status of coral reefs of the world: 2004. Queensland, Australian Institute of Marine Science, Townsville.

Yánez, A. P. (1997). Seminario Bibliográfico "Distribución de especies vegetales en ecosistemas naturales desde la óptica del Continuum: Evolución de la Idea y algunos Métodos para su Caracterización”. CIELAT, Universidad de Los Andes, Mérida, Venezuela, 44pp.

Yánez, P. (2005). Biometría y Bioestadística fundamentales. Analizando la estructura numérica de la información en proyectos ecológicos. Quito.

Yánez, P. (2014). Ecología y biodiversidad: un enfoque desde el neotrópico. Quito: UNIBE/UIDE.

Young, C., Schopmeyer, S., \& Lirman, D. (2012). A review of reef restoration and coral propagation using the threatened genus Acropora in the Caribbean and Western Atlantic. Bulletin of Marine Science, 88(4), 1075-1098. 